\title{
Mobbing: modelos explicativos y acción sindical
}

\author{
Susana Mayoral Blasco \\ Universidad de Zaragoza. Departamento de Psicología y Sociología \\ smayoral@unizar.es
}

Recibido: 10-06-2008

Aceptado: 16-02-2009

\section{Resumen}

El mobbing se ha convertido en un riesgo psicosocial emergente en el mundo laboral actual, una realidad ante la cual diversas instancias intentan reaccionar, promoviendo su estudio y realizando propuestas preventivas. En el presente texto, se valoran varios de los modelos teóricos actualmente utilizados para dar cuenta del mobbing, resaltando sus virtudes y limitaciones, con el objetivo de proponer un nuevo modelo de análisis basado en la teoría de la acción práctica, de Pierre Bourdieu. Mediante un estudio de caso, se muestra cómo este nuevo modelo es capaz de integrar muchas de las aportaciones de los demás modelos, al tiempo que permite una mayor profundidad de análisis y tiene más capacidad explicativa. Se incluye también un protocolo de análisis para casos de mobbing basado en el modelo propuesto.

Palabras clave: poder, Pierre Bourdieu, modelos teóricos, riesgos laborales.

\section{Abstract. Mobbing: explanatory models and labour union's action}

Mobbing has turned into an emergent psychosocial risk in the current world of work. It is a real fact in the face of which different institutions and social agents try to react, promote its study, and carry out preventive proposals. Several theoretical models used to account for mobbing are here analysed, and their virtues and shortcomings are highlighted, with the aim of suggesting a new model of analysis based on Pierre Bourdieu's theory of practical action. It is shown, through a case study, how this new model is capable of integrating many of the contributions of the other models, while allowing for deeper analysis and greater explanatory power. In addition, the article includes a protocol for the analysis of mobbing cases, which is based on the suggested model.

Key words: power, Pierre Bourdieu, theoretical models, risks at workplace. 


\author{
Sumario \\ 1. Introducción 5. Resultados \\ 2. Descripción y valoración de los \\ 6. Conclusiones \\ modelos más utilizados \\ Referencias bibliográficas \\ 3. Modelo propuesto \\ Anexo 1
}

4. Metodología y técnicas

\title{
1. Introducción
}

En este articulo, presentamos un modelo teórico para analizar el mobbing basado en la teoría de la acción práctica de Pierre Bourdieu y que, además, intenta recoger las aportaciones de los modelos de análisis usados más habitualmente por la acción sindical en España (modelo psicopatológico, Iñaki Piñuel, 2001; modelo de la psicología social, Jorge Corsi y Graciela Peyrú, 2003, y modelo sociolaboral, Dolores Fernández y Clara Llorens, 2002), a la vez que constituir una superación de los mismos ${ }^{1}$. El origen del modelo que proponemos hay que situarlo en una investigación sobre mobbing y acción sindical (Mayoral, 2003) donde se analizaban dos casos de mobbing, uno en una empresa pública de ámbito estatal y otro en una administración pública local. En el análisis de los casos, se detectó que los actores implicados (víctimas, sindicatos y quienes los encubren) se confrontaban en diferentes arenas (empresa, tribunales, medios de comunicación) utilizando diferentes recursos. Estos recursos comprendían el poder formal e informal, la ley o la capacidad de atacar a la imagen pública del oponente, entre otros. Aparecían también como factores de peso, en el desarrollo del conflicto, la cultura de acoso de la empresa y la cultura sindical. Todo ello nos proporcionaba una visión del mobbing como la de un conjunto de actores (agentes) que luchaban en diferentes arenas (lo que nos remitía al concepto de campo de Bourdieu), utilizando para ello todos los recursos a su disposición (lo que nos remitía al concepto de capital, social, simbólico, cultural y económico); con una manera de hacer propia de cada uno de los actores que comprendía comportamientos, estrategias y una serie de razones y valores que los sustentaban, entre ellos, el de ser práctico (lo que nos remitía al concepto de habitus).

También pudimos observar que los modelos explicativos específicos del mobbing (Piñuel, 2001; Corsi y Peyrú, 2003; Fernández y Llorens, 2002) eran válidos, puesto que sí contribuían a explicar los casos, pero también parciales, puesto que privilegiaban determinados factores y dejaban a otros que parecían ser importantes en un segundo plano. Otro de los resultados de esta investigación es que ninguno de los modelos incorpora de forma que consideremos

1. Este modelo teórico constituye el resultado final de una investigación realizada entre los años 2003 y 2007, en el marco del doctorado en Sociología de la Universidad Autónoma de Barcelona. 
adecuada factores como el poder o la cultura de la empresa, que parecen ser claves en el desarrollo del mobbing.

Por todo ello, parecía existir la necesidad de aplicar un modelo teórico más macroestructural, capaz de dar cuenta del fenómeno en toda su complejidad y no sólo de algunos aspectos, pero que también fuera capaz de incorporar plenamente al análisis los factores del poder y la cultura de la empresa. En el presente artículo, vamos a revisar estos modelos y a presentar nuestra propuesta.

\section{Descripción y valoración de los modelos más utilizados}

\section{a) El modelo psicopatológico}

El psicólogo Iñaki Piñuel, a raíz de la publicación, en 2001, de su libro Mobbing: Cómo sobrevivir al acoso psicológico en el trabajo, se convirtió en uno de los principales impulsores de este modelo, que ha tenido una importante influencia en la definición de esta problemática en el mundo laboral español. Este autor define el mobbing como:

El acoso tiene como objetivo intimidar, apocar, reducir, aplanar, amedrentar y consumir emocional e intelectualmente a la víctima, con vistas a eliminarla de la organización o de satisfacer la necesidad insaciable de agredir, controlar, destruir que suele presentar el hostigador, quien aprovecha la situación que le brinda la situación organizativa particular (reorganización, reducción de costes, burocratización, cambios vertiginosos, etc.) para canalizar una serie de impulsos y tendencias psicopáticas. (Piñuel, 2001: 55)

De manera genérica, este modelo tiende a centrar el estudio del mobbing en la identificación de los perfiles del acosador (que sufre una serie de trastornos psicológicos, como pueden ser personalidad paranoide, psicópata o narcisista), que coincide por azar en el ámbito laboral con una persona cuyas características personales altamente positivas (persona brillante en el trabajo, o con éxito en la vida afectiva o personal en general) activan las conductas propias de estas patologías. Desde esta perspectiva psicopatológica, se admite que, en cierto modo, la organización del trabajo actúa como contexto favorecedor del acoso, pero se sigue poniendo el acento en la relación interpersonal y se ha tendido a buscar y a elaborar «perfiles» del acosador y de la víctima.

Algunos autores de referencia en el tema, como el psicólogo Jesús Pérez Bilbao y otros (2001) o la psiquiatra Marie France Hirigoyen (2001), cuestionan la idea del "perfil de víctima" y defienden que cualquiera, en cualquier momento de su vida laboral, puede ser víctima del mobbing. Por su parte, Concepción Sáez Navarro (2001) plantea que, en los estudios realizados, no ha sido posible encontrar un perfil típico ni del acosador (mobber) ni de la víctima (mobbed), sin embargo, se suele estar de acuerdo en considerar que el acosador percibe a la víctima como peligrosa por diferentes razones. En todo caso, como consecuencia de esta construcción del mobbing como la de una especie de juego psicológico destructivo entre acosador y víctima, rodeados de testi- 
gos mudos, el acoso se acaba cuando uno de los jugadores (la víctima) «deja de jugar al juego». De esta construcción del problema, se derivan las recomendaciones de Piñuel (2001) e Hirigoyen (2001), quienes, tras dar una serie de recomendaciones sobre cómo enfrentar el acoso en libros del tipo manual de autoayuda, recomiendan que, en caso de que no se posean los recursos personales suficientes, es necesario acudir a un profesional de la psicología. Esta lectura del problema, que fue la que predominó durante los años 2001-2002, dio lugar a una medicalización del mismo y también a una individualización, con lo que el tipo de medidas que se tomaban para la resolución de los casos solían ser la de una baja médica prolongada para la víctima cuando empezaba a manifestar los síntomas típicos de ansiedad y depresión, y, en el mejor de los casos, un cambio de puesto de trabajo en aquellas situaciones en las que la víctima no optara por abandonar la empresa, sino por reincorporarse a la misma (caso frecuente de los funcionarios, por ejemplo).

\section{b) El modelo de la psicología social}

Jorge Corsi y Graciela Peyrú (2003), a partir de las definiciones de otros autores, pero fundamentalmente de Hirigoyen (2001), elaboran la siguiente definición del mobbing:

El concepto agrupa una diversidad de comportamientos que tienen una finalidad común: la modalidad agresiva y vejatoria con o sin intención de eliminar a un trabajador de la empresa. Las formas en las que suele presentarse son variadas y de las más sutiles: el aislamiento físico del trabajador; la marginación a través de la hostilidad y la falta de comunicación; la difusión de comentarios peyorativos; la asignación de tareas humillantes, descalificadoras o de muy difícil concreción; el menoscabo de la imagen del empleado frente a sus colegas, clientes o superiores; los cambios continuos; etc. El impacto de este abuso emocional puede ser definitivo cuando destruye la autoconfianza del acosador en su competencia o en su sentido de los hechos. Todas estas modalidades de acoso al trabajador, obviamente, no constituyen por sí mismas ninguna novedad. Lo que sí concita la atención de los juristas es el análisis del problema sobre la base de la repetición de estos hechos, en un lapso suficientemente prolongado y la relación asimétrica entre el agresor y la víctima ${ }^{2}$. (Corsi y Peyrú, 2003: 183)

La originalidad de estos autores reside en incluir en su obra Violencias sociales (2003) la violencia en el trabajo y, dentro de ésta, el mobbing, a consecuencia de lo cual queda incorporado el tema del poder al análisis. Además, lo conceptualizan como violencia laboral (resultado de las dinámicas de violencia colectiva que forman parte de la cultura de la organización laboral), diferenciándola de la violencia en el trabajo (que haría referencia a un asalto a mano armada en el lugar de trabajo, por ejemplo). En este sentido, plantean que los

2. Las cursivas son las del original. 
mismos mecanismos presentes en otros tipos de violencia social, que permiten su aparición y reproducción (la invisibilización de la violencia, su naturalización, la insensibilización de quienes la presencian o participan en ella y el encubrimiento), actúan también en la aparición y reproducción del mobbing en una organización laboral.

Como principales aportaciones del modelo, hemos de subrayar la de ampliar la visión del mobbing del modelo psicopatológico centrada en el binomio formado por el acosador y la víctima, para plantear que el mobbing es un producto colectivo. Otra de sus aportaciones radica en que, al conceptualizar el mobbing como una violencia social, abre la posibilidad de aplicar a su estudio las mismas herramientas conceptuales que se aplican al estudio de otro tipo de violencias, lo cual amplía las posibilidades de conocer el fenómeno en mayor profundidad y desde otra perspectiva, especialmente cómo se produce y se reproduce como parte de las dinámicas colectivas habituales en la organización laboral. Como última aportación, diremos que liga los conceptos de violencia, poder y cultura al análisis del mobbing.

\section{c) La perspectiva sociolaboral}

Dolores Fernández y Clara Llorens (2002) establecen una relación entre la organización del trabajo y los daños a la salud (concepto de riesgos psicosociales) y elaboran un modelo explicativo específico del mobbing. Estas autoras definen el mobbing poniéndolo en relación con el contexto laboral. En concreto, la definición que dan es la siguiente:

Entonces ¿qué es el acoso moral? Es un riesgo laboral derivado de las condiciones de trabajo, cuyos efectos en la salud son daños derivados del empleo y es una obligación empresarial prevenirlo. Se produce cuando el empresario o sus representantes o cualquier trabajador(es) que tiene(n) una posición de mayor poder (reconocido como una categoría laboral superior, o de facto, por tener mayores apoyos, mayor antigüedad, etc.), actúan con el propósito de dañar a otro/a u otros/as trabajadores usando las deficiencias en la organización del trabajo de forma repetida y frecuente en el tiempo. Lo que diferencia el acoso moral de otros problemas derivados de la organización del trabajo es la intencionalidad de causar daño, la focalización en una o varias personas, la repetición, la frecuencia y la continuidad en el tiempo. Si no se dan estas características, hablaremos de factores de riesgo psicosocial derivados de la organización del trabajo, pero no estaremos hablando de un caso de acoso moral. (Fernández y Llorens, 2002: 2)

A partir esta conceptualización del acoso, elaboran el siguiente modelo desarrollado en la figura 1 .

Estas autoras defienden que las deficiencias en la organización del trabajo y la intencionalidad de causar daño son la base necesaria para que podamos hablar de acoso. De todos modos, el elemento fundamental es la organización del trabajo, puesto que si hay intención de causar daño pero la organiza- 
Figura 1. Modelo sociolaboral

\begin{tabular}{|c|c|c|c|c|}
\hline $\begin{array}{l}\text { Factores de riesgo psicosociales } \\
\text { Falta de control, influencia, } \\
\text { claridad de rol, previsibilidad, } \\
\text { inseguridad... } \\
\text { (derivados de la organización } \\
\text { del trabajo) } \\
+ \\
\text { Falta de apoyo social } \\
\text { (Instrumento de medida ISTAS 21) }\end{array}$ & + & $\begin{array}{l}\text { Intención } \\
\text { Frecuencia } \\
\text { Focalización } \\
\text { Sutileza }\end{array}$ & $=$ & $\begin{array}{l}\text { Ansiedad } \\
\text { Depresión } \\
\text { Insomnio } \\
\text { Aislamiento del mundo } \\
\text { Pérdida de la autoestima }\end{array}$ \\
\hline Acoso & & & & Daños a la salud \\
\hline
\end{tabular}

Fuente: Fernández y Llorens, 2002: 3.

ción laboral no puede ser usada como elemento hostigado, no se dará el acoso. Eso significa también que la personalidad del acosador o de la víctima no son necesariamente la base del problema (Fernández y Llorens, 2002: 3). El núcleo central de este modelo es mostrar como los riesgos psicosociales actúan como caldo de cultivo que facilita la aparición del mobbing, pero también pueden ser usados como herramienta de acoso, siendo la actuación intencionada del acosador la que los convierte de factor de riesgo a un instrumento de acoso, al someter a la víctima a una exposición focalizada, frecuente y prolongada a los mismos. Al igual que una exposición focalizada, frecuente y prolongada a un factor de riesgo físico o químico genera daños físicos a la salud, una exposición de estas características a riesgos psicosociales produce daños a la salud psíquica (síndrome de ansiedad y depresión), pero también daños a la salud física (taquicardia, dificultades respiratorias, etc.). Estas autoras, además de proponer un modelo explicativo del mobbing realizan una serie de propuestas generales de prevención e intervención dirigidas a orientar la acción sindical.

\section{d) Valoración general de los modelos}

La valoración general que hacemos de estos modelos es que son válidos, puesto que todos ellos contribuyen a conocer el fenómeno, pero, al mismo tiempo, son parciales. Como hemos podido ver, cada uno de ellos explica el mobbing privilegiando determinados factores, como son los perfiles personales en el caso del modelo psicopatológico (Piñuel, 2001), las dinámicas de violencia grupal en el modelo psicosocial (Corsi y Peyrú, 2003) o la existencia de riesgos laborales en el modelo sociolaboral (Fernández y Llorens, 2002). Pero consideramos que todos ellos incorporan demasiado débilmente factores que parecen ser importantes, como el poder y la cultura de la empresa.

Respecto al poder, hemos podido ver que todos los modelos reconocen la centralidad del poder en la existencia del mobbing, pero lo sitúan fuera del 
modelo, es decir, estos modelos parecen construirse de la siguiente manera: partiendo de que existe una relación asimétrica de poder entre el acosador y la víctima, vamos a explicar por qué se produce el mobbing. Consideramos que ésta es una deficiencia grave de todos los modelos. A partir de ello, podemos plantearnos las siguientes preguntas: si la relación asimétrica de poder está en la base del mobbing, ¿no es posible que el acoso disminuya o cese en la medida en que esta relación asimétrica sea alterada o desaparezca?; si la gestión del conflicto por parte de los superiores parece ser un elemento importante en el desarrollo de los casos (NTP 476, Martín Daza y otros, 1998), ¿no tendrán algo que ver en el tipo de intervención que se hace la posición de poder que ocupa el acosador y la víctima respecto a quienes tienen que intervenir (los superiores)?3. Por lo tanto, nuestra propuesta es que, puesto que es un factor clave reconocido como tal por todos los autores y autoras en sus definiciones, sería necesario integrar el poder dentro del análisis del mobbing, y dentro de cualquier modelo explicativo del mismo.

Como segunda carencia común a todos los modelos, señalamos que, aunque todos ellos intentan vincular el contexto con la aparición y el desarrollo del mobbing, en realidad cada uno de los modelos incorpora aspectos parciales de este contexto, por lo que todos ellos dejan fuera factores que podrían ser relevantes. Así, el modelo psicopatológico de Piñuel (2001) menciona las formas de organización del trabajo y el comportamiento organizativo como factores situacionales que facilitan la aparición del mobbing ${ }^{4}$, pero el factor explicativo sobre el que se articula su modelo es el del perfil personal, tanto del acosador como de la víctima. El modelo de la psicología social de Corsi y Peyrú (2003) conecta más el mobbing con el contexto, pero la relación consiste en conectar el caso de mobbing con la dinámica grupal de violencia que forma parte de las dinámicas laborales de la organización donde se da el caso de mobbing. Y, finalmente, el modelo de Fernández y Llorens (2002) realiza una conexión con el contexto, al relacionar el mobbing con la organización del trabajo, concretamente, con los riesgos psicosociales. De alguna manera, todos ellos intentan conectar el mobbing con la organización del trabajo (Fernández y

3. Fundamentamos la pertinencia de esta pregunta en que, según los datos aportados por el Barómetro Cisneros II (Piñuel y Oñate, 2002), en el 82,13\% de los casos, las victimas refieren que son acosadas por los jefes.

4. En una obra posterior, Neomanagement: Jefes tóxicos y sus víctimas (Piñuel, 2005), concede una mayor atención a las dinámicas de la empresa. En esta obra, el autor dedica una mayor extensión a describir el entorno organizacional, refleja de alguna manera el cambio que ha experimentado el mundo laboral de la década de 1990 y describe algunas organizaciones laborales como tóxicas, puesto que se empieza a implantar un nuevo estilo de gestión de los recursos humanos que incluye la violencia psicológica, las guerras de todos contra todos, la feudalización de las relaciones laborales, etc., todo ello amparado por el nuevo contexto de precariedad laboral y globalización económica. Sin embargo, aunque se da una mayor cabida a elementos culturales y a dinámicas organizativas, el autor los aborda de una forma demasiado descriptiva, sin profundizar en ellos, ya que son el telón de fondo para los comportamientos analizados, pues vuelve a centrarse en comportamientos individuales, como anuncia claramente la segunda parte del título de su obra (Jefes tóxicos y sus víctimas). 
Llorens, 2002) o con la cultura de la empresa (Corsi y Peyrú, 2003), pero ninguno lo hace con los dos aspectos a la vez. El único modelo que, de alguna manera, menciona los dos a la vez es el de Piñuel (2001), pero únicamente hace eso, mencionarlos como telón de fondo, pues no vuelve sobre ellos en ningún momento a lo largo de su obra.

\section{Modelo propuesto}

Todo lo anterior nos lleva a plantear la necesidad de un modelo explicativo específico del mobbing, que incorpore estos factores, el poder, el contexto y la cultura, no como telón de fondo, sino como factores explicativos de peso. Proponemos como modelo provisional capaz de integrar dentro estos factores, así como los aportados por los modelos revisados, el modelo de la teoría de la acción de Pierre Bourdieu (1998, 2002), donde:

\section{PRÁCTICA $=[$ CAMPO + (CAPITAL $)($ HABITUS $)]$}

Dado que este modelo ha demostrado su potencia para explicar otras realidades sociales, sugerimos que también es aplicable al análisis del mobbing. Por ello, la originalidad de nuestra propuesta no reside tanto en elaborar un modelo nuevo, sino en abordar el análisis del mobbing desde una perspectiva sociológica basada en esta teoría. A continuación, presentamos gráficamente una primera adaptación de la formulación de Bourdieu $(2002,1998)$ al estudio del mobbing, a modo de hipótesis de partida.

Figura 2. Aplicación del modelo de la teoría de la acción de Bourdieu al análisis del mobbing

\begin{tabular}{|c|c|c|c|c|c|}
\hline Práctica & $=$ & Campo & + & [(Capital & (Habitus)] \\
\hline$\downarrow$ & & $\downarrow$ & & $\downarrow$ & $\downarrow$ \\
\hline$\forall$ & & $\begin{array}{c}\searrow \\
\text { Los agentes }\end{array}$ & & $\checkmark$ & Cultura de \\
\hline Mobbing & $=$ & $\begin{array}{c}+ \\
\text { Las prácticas }\end{array}$ & & Poder & la empresa \\
\hline
\end{tabular}

Fuente: elaboración propia.

\section{Metodología y técnicas}

Para contrastar empíricamente nuestra propuesta, es decir, la utilidad y la parcialidad de los modelos de Piñuel (2001), Corsi y Peyrú (2003) y Fernández y Llorens (2002), a la vez que la mayor potencia explicativa de nuestro modelo teórico, procedemos a aplicar todos los modelos al análisis de un mismo caso y a comparar los resultados respecto a la información y a la comprensión que aporta cada uno de ellos. 
Para contrastar la potencia explicativa de los modelos, se eligieron dos casos, uno en el ámbito público y otro en el ámbito privado, que se fueron investigando en paralelo. A medida que iba avanzando la investigación, pudimos observar que, aunque había dinámicas comunes (la organización del trabajo como instrumento de acoso o la implicación activa de la cadena de mando), el caso del ámbito público tenía unas características específicas que lo diferenciaban, como era la importancia en el mobbing de las lógicas políticas frente a las lógicas económicas propias del ámbito privado, aspecto éste que ya había sido apuntado por Hirigoyen (2001: 110) y que ya habíamos detectado en investigaciones previas (Mayoral, 2003).

Por tanto, tras comprobar de forma exploratoria en el caso de la empresa privada la capacidad explicativa de los diferentes modelos, decidimos centrarnos en el estudio de uno solo, el de la empresa pública de ámbito estatal, a fin de poder estudiarlo con mayor profundidad. Lo hacemos porque, además de detectar que estos casos tienen estas características específicas, a partir de la revisión bibliográfica, comprobamos que existe un gran vacío de conocimiento sobre casos de mobbing en la Administración o en las empresas públicas, con una casi inexistencia de estudios cualitativos (con la excepción de Miguel Ángel Sabio Checa, 2003, desde su perspectiva de técnico superior en prevención de riesgos laborales).Por lo tanto, nos proponemos aprovechar la oportunidad de estudiar un caso aplicando diferentes modelos teóricos, para elegir un ejemplo de un tipo poco estudiado.

Somos conscientes de que los resultados obtenidos de estudiar un solo caso no se pueden generalizar a toda la Administración pública. Lo único que nos proponemos, como un objetivo secundario, es generar conocimiento sobre casos de mobbing en la Administración pública y, por lo tanto, aportar conocimiento sobre uno solo, ya supone un incremento del mismo (Flyvbejerg, 2004). Pero recordamos que nuestro objetivo es contrastar la potencia explicativa de los diferentes modelos teóricos revisados y del modelo propuesto.

Como técnicas, se han usado, en primer lugar, la recogida y el estudio de documentación, en concreto: informes de Inspección de Trabajo, sentencias judiciales, la evaluación de riesgos laborales de la empresa elaborada por su mutua, denuncias de diversos delegados sindicales y sindicatos a Inspección de Trabajo, escritos de la sección sindical de la empresa a la empresa (con firmas manuscritas y sello del registro de entrada de la empresa), respuestas de las jefaturas (con firma manuscrita y sello), denuncias a la guardia civil (con número de registro y sello de la comandancia), escritos al defensor del pueblo de la comunidad autónoma, escritos al defensor del pueblo de España, así como la respuesta, además de variada documentación interna de la empresa relacionada con el caso. En total, cincuenta y ocho documentos, la mayoría de ellos oficiales, ya que el caso ha pasado varias veces por la vía judicial.

También se realizaron entrevistas en profundidad abiertas en la fase exploratoria y entrevistas semiestructuradas en la fase de recogida de la información a diversos informantes. Finalmente, se recogió material gráfico (prensa, fotografías y grabaciones televisivas), todo él relacionado con el caso. La 
diversidad y el gran volumen de información generada, permitía la triangulación de la misma. Una de las limitaciones de la investigación es que no se pudo entrevistar a los acosadores ni a las jefaturas, algo habitual en las investigaciones sobre mobbing. Consideramos, sin embargo, que la triangulación es posible, puesto que la gran cantidad de información existente suple esta carencia.

\section{Resultados}

Como resultado del estudio de caso, pudimos comprobar varios aspectos relevantes que, a continuación, se detallan.

En primer lugar, se observa que la aplicación del modelo psicopatológico de Piñuel (2001) no puede explicar satisfactoriamente el caso, ya que no se puede comprobar el perfil de psicópata, paranoico o narcisista de los acosadores. Es, por tanto, el modelo de menor capacidad explicativa. En cambio, el modelo de la psicología social de Corsi y Peyrú (2003) resulta muy útil para el estudio de la cultura de la empresa relacionada con el acoso, ya que nos permite detectar cuales son los procesos psicosociales facilitadores de la violencia presentes en la empresa, que permiten que el mobbing se produzca y se reproduzca (naturalización, encubrimiento, premiar a quienes ejercen el mobbing y castigar a quienes defienden a la víctima, etc.), pero no da cuenta de otros factores presentes en la situación.

Por otro lado, el modelo de Fernández y Llorens (2002) nos permite identificar en el caso como, efectivamente, se expone de forma continua y prolongada a la víctima a los riesgos psicosociales (aislamiento, falta de claridad de rol, falta de feedback, feedback constantemente negativo), y como esto produce daños a su salud que se manifiestan en un síndrome de ansiedad-depresión, con las bajas médicas consiguientes. Es un modelo útil para justificar la intervención sindical en el caso amparada en la Ley de prevención de riesgos laborales 31/1995, de 8 de noviembre, pero insuficiente para explicar el mismo en toda su complejidad.

Por último, al aplicar el modelo que proponemos, obtenemos los siguientes resultados. Por un lado, nos proporciona más información de la necesaria para contrastar las hipótesis específicas planteadas para explicar el caso. Y, por otro lado, tiene unos conceptos lo suficientemente potentes y abiertos (campo, capital, habitus) y unas dimensiones lo suficientemente flexibles (agentes, estructura del campo, prácticas en el campo) como para poder incluir tanto los factores contemplados por los otros modelos, como los factores que no son considerados por ninguno de los modelos anteriores, pero que, sin embrago, aparecen como factores de peso a medida que se desarrolla la investigación de un caso concreto.

Tras explicar las potencialidades de cada uno de los conceptos y del modelo en su conjunto para el análisis del mobbing, presentamos seguidamente una primera operacionalización del mismo (ver figura 3). Se trata de una especie de plantilla, que pueda actuar a modo de protocolo o guía para el análisis de 
Figura 3. Esquema general del modelo



Fuente: elaboración popia

casos de mobbing concretos desde la acción sindical o la acción desarrollada por técnicos en prevención de riesgos laborales 5 .

Éste sería probablemente el principal punto débil del modelo, su falta de aplicabilidad práctica inmediata, ya que, por ejemplo, no existen instrumentos de medida estandarizados de las relaciones de poder informales o de la cultura de la empresa respecto al mobbing. Ésta es una debilidad del modelo, especialmente frente al modelo sociolaboral de Fernández y Llorens (2002), que cuenta con su propio instrumento de medida, el test ISTAS 21. Sin embargo, consideramos que puede tratarse de una debilidad temporal y que puede actuar como una propuesta teórica en la que basar la creación de nuevos instrumentos destinados a la prevención e intervención en casos de mobbing desde la acción sindical.

El concepto de campo nos permite incluir el contexto como un factor de peso en la existencia del mobbing. El campo consta de tres dimensiones: agentes, estructura del campo y prácticas del campo, que nos permiten operacionalizar el análisis del contexto.

La dimensión de agentes nos permite incorporar especialmente las aportaciones de Piñuel (2001) al estudio del mobbing, ya que este autor considera

5. Dicha plantilla se puede consultar en el anexo 1 . 
como el factor determinante en el mobbing, los perfiles personales del acosador y de la víctima, rodeados de una serie de testigos mudos. Pero el concepto de agentes de Bourdieu es más amplio y nos permite superar esta visión e incorporar otro tipo de actores, internos y externos, como la cadena de mando (NTP 476: 1998), las secciones sindicales y los sindicatos (Fernández y Llorens, 2002), el Sistema Judicial o Inspección de Trabajo, cuya actuación tiene una indudable influencia en el desarrollo de los casos.

La dimensión de estructura del campo nos permite realizar una primera integración del factor poder al análisis, lo cual era una de las deficiencias de los modelos que pretendíamos subsanar, ya que nos permite situar a los actores como nodos de una red de relaciones de poder formales y, quizá lo que parece ser muy importante, informales.

Las redes de poder informales parecen tener un gran peso en todos los casos de mobbing analizados, y la subdimensión de estructura informal del campo nos permite realizar el análisis de las mismas. Pero quizá la mayor potencialidad de la estructura del campo es que nos permite entender el mobbing como un medio (una práctica) utilizado por aquellos agentes que poseen el poder (formal, informal o ambos) en el campo, para mantener su posición de poder eliminando a quien consideran que la ataca. Es decir, dado que, según Bourdieu, la estructura del campo es inestable, porque es producto de la relación de fuerzas entre los agentes presentes en él, podemos entender el mobbing como una práctica desarrollada por los dominantes para mantener su posición en el campo $y$, a su vez, mantener intacta la estructura del campo. Esta lectura del mobbing también nos permite entender la implicación de la cadena de mando apoyando y encubriendo a los acosadores. Puesto que forman parte de los dominantes, al apoyar y encubrir al acosador, no hacen más que defender la estructura del campo existente y, más probablemente, su propia posición en la misma.

Esta interpretación del mobbing como práctica para defender la estructura del campo y su posición por parte de los dominantes, también nos permite entender por qué, aunque existan medidas explicitadas y formales para intervenir en los casos de mobbing en la empresa (protocolos específicos de intervención, artículos del convenio colectivo), éstas no se aplican. Porque el aplicarlas (por ejemplo: sancionar a un jefe y dar la razón a un trabajador) supone alterar las relaciones de poder entre dominantes y dominados en la empresa y, por lo tanto, alterar la estructura del campo.

La dimensión de las prácticas del campo nos permite integrar aquellas aportaciones sobre las prácticas formales e informales en relación con la organización del trabajo y con las relaciones laborales, que pueden actuar como caldo de cultivo del mobbing (NTP 476, 1998; Piñuel, 2001; Fernández y Llorens, 2002). Pero además de las prácticas descritas por estos autores, nos permite integrar todas aquéllas no descritas y específicas del caso analizado.

El concepto de capital nos permite integrar la aportación de Fernández y Llorens (2002), que consideran los riesgos psicosociales como una herramienta para ejercer el acoso, luego, en nuestros términos, podemos considerarlo un capital. Pero la gran aportación del concepto de capital al análisis del mobbing 
es que nos permite integrar, de manera plena y como factor de peso, el factor poder. Este concepto nos permite subsanar uno de los déficits que atribuíamos a los demás modelos, que decíamos parecían construirse de la siguiente manera: a partir de que existe una relación de poder, vamos a explicar por qué se da el mobbing. Integrar el factor poder nos puede llevar a entender el mobbing como un modo particular de ejercer el mismo, es decir, el mobbing entendido como manera de ejercer el poder en su forma más extrema, mediante la violencia, ya que se trata de causar daño o de eliminar a la víctima.

En la investigación realizada, pudimos comprobar la centralidad del poder en la aparición y el mantenimiento del mobbing, hasta el punto de que una disminución en el poder del acosador supone una disminución o incluso el cese del acoso. Esto abre una puerta a la intervención, dado que muchos de los casos se «resuelven» cambiando a la víctima de puesto de trabajo. En estos casos no se cambian las condiciones estructurales para que el acoso no se vuelva a repetir, que tampoco pasarían por cambiar de puesto al acosador, sino por dejarlo sin capital para ejercerlo, es decir, tomar las medidas necesarias para dejarlo sin poder (degradación funcional, despido).

Evidentemente, se pueden tomar con mayor facilidad medidas destinadas a la disminución del poder formal, ya que el poder informal de los agentes es de difícil control. Para controlar el poder informal de los acosadores, quizá sería necesario tomar otras medidas, como un proceso de criminalización de los mismos y de estas prácticas.

El concepto de habitus es también una de las grandes aportaciones del modelo al análisis del mobbing. Este concepto nos permite integrar la cultura de la empresa al análisis, factor clave según varios autores (Agencia Europea para la Seguridad y la Salud en el Trabajo, 2002; Pérez Bilbao, 1998, 2001, 2002; Sáenz Navarro, 2001). Nos permite integrar especialmente las aportaciones de Corsi y Peyrú (2003), quienes conciben el mobbing como resultado de las dinámicas grupales de violencia existentes en la organización laboral.

La potencia del concepto de habitus reside en que su dimensión de habitus como estructura estructurada nos permite detectar como la cultura de acoso individual y colectiva existente en la empresa influye en la aparición del acoso. A su vez, su dimensión de habitus como estructura estructurante nos permite identificar por qué mecanismos esta cultura del acoso (y el propio acoso) se reproducen en la empresa.

En la investigación realizada, el concepto de habitus resulta fundamental para entender el mobbing. Frente a la habitual consideración del mobbing como una disfunción del sistema, el concepto de habitus nos permite plantearnos que si el mobbing se produce y se reproduce en una organización laboral, es porque es funcional (en términos de Bourdieu, diríamos que es práctico). Es funcional (o práctico) cuando, sin apenas costes (no se sanciona), permite obtener importantes beneficios (como mantener la posición de poder y los beneficios materiales o simbólicos que de ella se derivan). Ello abre las puertas a la intervención, ya que una manera de intervenir sobre el mobbing es llevar a cabo actuaciones que hagan que éste deje de ser funcional, lo que influiría tanto en 
su práctica individual como en su práctica colectiva, como parte de la cultura de la empresa.

\section{Conclusiones}

En definitiva, la aplicación del modelo de la teoría de la acción de Bourdieu, donde Práctica $=$ Campo + [(Capital) $($ Habitus $)]$, al análisis del mobbing, nos permite, en primer lugar, realizar un análisis sociológico del mismo.

También nos ha permitido obtener un modelo de análisis que permite integrar conocimiento previo generado por otros modelos anteriores. Ello es posible porque los conceptos de Campo, Capital y Habitus son lo suficientemente potentes y flexibles como para poder encuadrar dentro de ellos factores que otros modelos señalaban como importantes en el desarrollo del mobbing, como los riesgos psicosociales (Fernández y Llorens, 2002) o las dinámicas de violencia social presentes en la empresa, por ejemplo (Corsi y Peyrú, 2003).

Asimismo, estos conceptos nos permiten incorporar factores que varios autores de referencia en el tema señalaban como claves en el mobbing, como el factor poder (encuadrado dentro del concepto de capital) o el factor cultura de la empresa (encuadrado dentro del concepto de habitus), y que los modelos anteriores incorporaban demasiado débilmente, a la vez que incorporar todos aquellos factores específicos del caso que estemos analizando. Finalmente, el modelo nos proporciona una lectura más profunda del fenómeno, dando cuenta de él en mayor medida que los modelos explicativos previos.

\section{Referencias bibliográficas}

Agencia Europea para la Seguridad y la Salud en el Trabajo (2002). «Acoso moral en el trabajo». Hoja Informativa (FACTS), no 23. Bruselas.

Bourdieu, Pierre (1990). Sociología y cultura. México: Consejo Nacional para la Cultura y las Artes.

- (1991). El sentido práctico. Madrid: Taurus.

- (1996). Cosas dichas. Barcelona: Gedisa.

- (1998). La distinción. Madrid: Taurus.

- (2002). Lección sobre la lección. Barcelona: Anagrama.

- (2002). Razones prácticas sobre la teoría de la acción. Barcelona: Anagrama.

- (2003). Cuestiones de sociología. Madrid: Istmo.

Bourdieu, Pierre y WaCQUANT, Löic (1994). Per una sociología reflexiva. Barcelona: Herder.

CORSI, Jorge y PeYrú, Graciela Maria (2003). Violencias sociales. Barcelona: Ariel. FERNÁNDEZ, Dolores y Llorens, Clara (2002). Acoso moral (mobbing): Una dimensión del riesgo psicosocial. [en línea]. <http://www.ccoo.es/istas>.

FLYVBEJERG, Bent (2004). «Cinco malentendidos acerca de la investigación sobre el estudio de casos». Revista Española de Investigaciones Sociológicas, n ${ }^{\circ} 106$, abriljunio, p. 32-57.

«Ley de prevención de riesgos laborales 31/1995, de 8 de noviembre». BOE, $\mathrm{n}^{\circ} 269$, de 10 de noviembre. [en línea]. <http://www. mtas. es /insht/legislación>. 
Hirigoyen, Marie France (2001). El acoso moral en el trabajo. Barcelona: Paidós.

Martín Daza, Félix; Pérez Bilbao, Jesús y López García-Silva, Juan Antonio (1998). El hostigamiento psicológico en el trabajo: Mobbing. Madrid: Instituto Nacional de Seguridad e Higiene en el Trabajo. Ministerio de Trabajo y Asuntos Sociales. Nota técnica de prevención 476.

Mayoral Blasco, Susana (2003). Mobbing y acción sindical. Tesina presentada en el Departamento de Sociología de la Universidad Autónoma de Barcelona. Inédito. En depósito en la Biblioteca de Ciencias Sociales de la UAB.

- (2007). Mobbing: Modelos explicativos y acción sindical. Tesis doctoral presentada en el Departamento de Sociología de la Universidad Autónoma de Barcelona. Inédito. En depósito en la Biblioteca de Ciencias Sociales de la UAB.

Pérez BilbaO, Jesús; Nogareda CuiXart, Clotilde; Martín Daza, Félix y Sancho FigUEROA, Tomás (2001). Mobbing, violencia física y acoso sexual. Madrid: Instituto Nacional de Seguridad e Higiene en el Trabajo. Ministerio de Trabajo y Asuntos Sociales.

Pérez BilbaO, Jesús (2002). Algunos apuntes sobre mobbing. [en línea]. <http://www.mtas.es/insht>.

Piñuel, Iñaki (2001). Mobbing: cómo sobrevivir al acoso psicológico en el trabajo. Santander: Sal Terrae.

- (2005). Neomanagement: Jefes tóxicos y sus víctimas. Madrid: Aguilar.

PIÑUEL, Iñaki y OÑATE, Araceli (2002). «La incidencia del mobbing o acoso psicológico en España. Resultados del barómetro Cisneros II sobre violencia en el entorno laboral». Lan Harremanak, vol. II, no 7, p. 35-62. Universidad del País Vasco.

SABIO CHECA, Miguel Ángel (2003). Violencia psíquica en una organización poco sensible a este problema: estrategias de actuación desde una perspectiva sindical. Inédito. En depósito en el Centro de Documentación del Centro Nacional de Condiciones de Trabajo (Barcelona). Ministerio de Trabajo y Asuntos Sociales.

SÁENZ NAVARRO, María Concepción (2001). «Algunas cuestiones sobre el mobbing en el trabajo. Comentario a la STSJ de Navarra del 30 de abril del 2001». Aranzadi Social, no 10 , p. 45- 51 . 


\begin{tabular}{l|l|l}
\hline Concepto & Variables & Dimensiones \\
\hline \multirow{2}{*}{ Agentes } & $\begin{array}{l}\text { Internos: agentes que forman parte de la plantilla de la empresa: acosador, testigos mudos, cómplices acti- } \\
\text { vos, cadena de mando, víctima, agentes colectivos que apoyan a la víctima (sección sindical, comité de } \\
\text { empresa). } \\
\text { Externos: agentes que no pertenecen a la plantilla de la empresa, pero cuyas actuaciones influyen en el } \\
\text { caso: psiquiatra o psicólogo, Inspección de Trabajo, agentes pertenecientes a otras instituciones, jueces, } \\
\text { sindicatos, etc. }\end{array}$ \\
\cline { 2 - 4 } & $\begin{array}{l}\text { Estructura formal: hace referencia a la red que forman los agentes implicados en el caso. Las posiciones } \\
\text { de los agentes en esa red dependen del poder formal que poseen éstos, derivado de la posición que ocupan } \\
\text { en el organigrama formal de la empresa o del poder formal que tienen los agentes de una institución res- } \\
\text { pecto a los agentes de otra institución, derivado de las posiciones formales que ocupan unas instituciones } \\
\text { respecto a otras (por ejemplo: Inspección de Trabajo y la empresa). } \\
\text { Incluye las posiciones de agentes individuales (por ejemplo: acosador y víctima) y de agentes colectivos } \\
\text { (por ejemplo: sindicatos y empresa). } \\
\text { Estructura informal: hace referencia a la existencia de redes informales y coaliciones. Estas redes o coaliciones } \\
\text { pueden ser internas, estar presentes sólo en el interior de la empresa y formadas por empleados; o trans- } \\
\text { versales, que unen una coalición interna con una externa (por ejemplo: redes formadas por empleados de } \\
\text { la empresa pública y por cargos políticos de instituciones públicas). La pertenencia a esas redes y coalicio- } \\
\text { nes dota de poder informal, y a su vez el poder informal individual previo a la pertenencia a esa red que } \\
\text { se posee, derivado del acceso a diferentes fuentes de poder informal, permite ocupar un lugar u otro en } \\
\text { dichas redes. }\end{array}$ \\
\hline
\end{tabular}


Anexo 1 (continuación)

\begin{tabular}{c|l|l}
\hline Concepto & Variables & Dimensiones \\
\hline Campo & $\begin{array}{l}\text { Prácticas } \\
\text { del campo } \\
\text { recogidas por escrito en el convenio, en los reglamentos internos, etc. Hacen referencia a la organización del } \\
\text { trabajo (mecanismos formales de organización del trabajo, funciones asignadas a cada puesto de trabajo, } \\
\text { duración de la jornada, turnos, etc.) y también a las relaciones laborales (estructura de la cadena de mando, } \\
\text { mecanismos de acceso al puesto de trabajo, mecanismos de ascenso, etc.). } \\
\text { Informales: conjunto de prácticas no explicitadas, porque algunas de ellas pueden no ser legítimas, que se } \\
\text { desarrollan en la empresa. Afectan a la organización del trabajo (por ejemplo: prácticas que generan riesgos } \\
\text { psicosociales) y a las relaciones laborales (por ejemplo: los traslados por procedimientos informales, incum- } \\
\text { plimiento de la normativa laboral respecto a contrataciones, descansos, jornada laboral, etc.). }\end{array}$ \\
\hline Poder & $\begin{array}{l}\text { Formal: factor derivado de la posición que se ocupa en el organigrama y que capacita para organizar el tra- } \\
\text { bajo, dar órdenes y que éstas hayan de ser cumplidas, premiar o sancionar. Cuanto más alto se está en el } \\
\text { organigrama, mayor es el poder formal y mayores son estas capacidades. Cuanto más bajo se está en el } \\
\text { organigrama, más disminuyen estas capacidades. } \\
\text { También se deriva de la ley (por ejemplo: el sindicato tiene poder formal para desarrollar determinadas } \\
\text { actuaciones en defensa de los trabajadores, amparado por la Constitución, el Estatuto de los Trabajadores } \\
\text { o la Ley de prevención de riesgos laborales). } \\
\text { Informal: proviene de numerosas fuentes, entre ellas, la pertenencia a redes y a coaliciones informales, las } \\
\text { alianzas con otros agentes que tienen poder, el control de la organización informal, de las contraorganiza- } \\
\text { ciones, del simbolismo y la dirección del pensamiento, de un recurso, de una habilidad técnica, de cono- } \\
\text { cimientos importantes para la organización, de los canales de información o del poder que ya se tiene. }\end{array}$ \\
\hline
\end{tabular}




\begin{tabular}{|c|c|c|}
\hline Concepto & Variables & Dimensiones \\
\hline \multirow{3}{*}{ Habitus } & $\begin{array}{l}\text { Cultura } \\
\text { colectiva }\end{array}$ & $\begin{array}{l}\text { Estructura estructurada: compuestas por aquellos elementos de la cultura de la empresa (presunciones } \\
\text { básicas, valores, normas de comportamiento, patrones de comportamiento, símbolos) que pueden actuar } \\
\text { como caldo de cultivo para la existencia del mobbing. Entre los patrones de comportamiento, se pueden } \\
\text { encontrar la presencia de una intensa actividad política, identificable por la existencia de un gran número } \\
\text { de juegos de poder (juego de construcción de imperios, juego para vencer a los rivales, juegos de rebeldía } \\
\text { y juegos de mando, que comprendería el mobbing). } \\
\text { Estructura estructurante: compuesta por aquellos elementos de la cultura de la empresa (presunciones } \\
\text { básicas, valores, normas de comportamiento, patrones de comportamiento y símbolos) que contribuyen a } \\
\text { producir y a reproducir el mobbing en la empresa. En los patrones de comportamiento, se puede detectar } \\
\text { la existencia de una serie de mecanismos facilitadores de la violencia, como son la negación de la violen- } \\
\text { cia, el encubrimiento de la violencia, la naturalización, la insensibilización de la violencia, definir con rigi- } \\
\text { dez los campos de lealtades y deslealtades, exaltar a quienes ejercen la violencia o minimizar los costos } \\
\text { humanos y materiales de ésta. }\end{array}$ \\
\hline & $\begin{array}{c}\text { Cultura } \\
\text { individual } \\
\text { (del } \\
\text { acosador/a) }\end{array}$ & $\begin{array}{l}\text { Estructura estructurada: hace referencia a los patrones de conducta del acosador en el ejercicio del mob- } \\
\text { bing. Incluye desarrollar las conductas de acoso calificadas como tales por la NTP 476, como ataques a la } \\
\text { víctima con medidas organizacionales, difusión de rumores, ataques a las actitudes y a los valores de la víc- } \\
\text { tima y amenazas del uso de la violencia. Puede incluir un gran repertorio de conductas destinadas a ani- } \\
\text { quilar psicológicamente a la víctima y conseguir su salida de la organización laboral. } \\
\text { Estructura estructurante: hace referencia a aquellas conductas desarrolladas por el acosador destinadas a } \\
\text { posibilitar su futuro ejercicio del mobbing (reproducción del mobbing). Incluye crear miedo entre los tes- } \\
\text { tigos mudos, premiar a quienes participan en el mobbing, perfeccionar sus estrategias de acoso, etc. }\end{array}$ \\
\hline & $\begin{array}{c}\text { Cultura } \\
\text { individual } \\
\text { (de la } \\
\text { víctima) }\end{array}$ & $\begin{array}{l}\text { Estructura estructurada: hace referencia a aquellos elementos de la cultura de la víctima que han podido } \\
\text { dificultar su defensa aunque sea víctima del mobbing (como, por ejemplo, un patrón de comportamiento } \\
\text { causado por el desconocimiento de que es el mobbing o dificultad para probarlo). } \\
\text { Estructura estructurante: hace referencia a aquellos elementos de la cultura de la víctima destinados a no } \\
\text { volver a ser víctima del mobbing. Incluye acudir al sindicato, ir a juicio, pedir que se den las órdenes por } \\
\text { escrito, etc. }\end{array}$ \\
\hline
\end{tabular}

